\title{
Global prognostic impact of driver genetic alterations in patients with lung adenocarcinoma: a real-life study
}

\author{
Panagiotis Paliogiannis ${ }^{1 \dagger}$, Maria Colombino ${ }^{2+}$, Maria Cristina Sini ${ }^{2 \dagger}$, Antonella Manca ${ }^{3}$, Milena Casula ${ }^{2}$, \\ Grazia Palomba², Marina Pisano ${ }^{3}$, Valentina Doneddu' ${ }^{1}$ Angelo Zinellu', Davide Santeufemia ${ }^{5}$, Sardinian Lung \\ Cancer (SLC) Study Group, Giovanni Sotgiu' ${ }^{4}$ Antonio Cossu ${ }^{1}$ and Giuseppe Palmieri ${ }^{3,4^{*}}$
}

\begin{abstract}
Background: Advanced lung adenocarcinoma $(L A C)$ is one of the most lethal malignancies worldwide. The aim of this study was to evaluate the global survival in a real-life cohort of patients with LAC harboring driver genetic alterations.

Methods: A series of 1282 consecutive Sardinian LAC patients who underwent genetic testing from January 2011 through July 2016 was collected. Molecular tests were based on the clinical needs of each single case (EGFRexon18/19/21, ALK, and, more recently, BRAF-exon15), and the availability of tissue (KRAS, MET, and presence of lowfrequency EGFR-T790M mutated alleles at baseline).

Results: The mean follow-up time of the patients was 46 months. EGFR, KRAS, and BRAF mutations were detected in $13.7 \%, 21.3 \%$, and $3 \%$ of tested cases, respectively; $A L K$ rearrangements and MET amplifications were found respectively in $4.7 \%$ and $2 \%$ of tested cases. As expected, cases with mutations in exons 18-21 of EGFR, sensitizing to anti-EGFR tyrosine kinase inhibitors (TKIs) agents, had a significantly longer survival in comparison to those without $(p<0.0001)$; conversely, KRAS mutations were associated with a significantly lower survival $(p=0.0058)$. Among LAC patients with additional tissue section available for next-generation sequencing (NGS)-based analysis, 26/193 (13.5\%) patients found positive for even low-rate EGFR-T790M mutated alleles at baseline were associated with a highly significant lower survival in comparison to those without (8.7 vs. 47.4 months, $p<0.0001$ ).

Conclusions: In addition to its predictive value for addressing targeted therapy approaches, the assessment of as more inclusive mutation analysis at baseline may provide clues about factors significantly impacting on global survival in advanced LAC patients.
\end{abstract}

Keywords: Lung adenocarcinoma, Mutation analysis, EGFR, KRAS, BRAF, ALK and MET rearrangements, Prognosis

*Correspondence: gpalmieri@uniss.it

${ }^{\dagger}$ Panagiotis Paliogiannis, Maria Colombino and Maria Cristina Sini contributed equally to this work

${ }^{3}$ Unit of Cancer Genetics, Institute of Genetic and Biomedical Research (IRGB), National Research Council (CNR), Traversa La Crucca 3, 07100 Sassari, Italy

Full list of author information is available at the end of the article

\section{Introduction}

Lung cancer is currently one of the most incident and lethal malignancies; in accordance with data from the Global Cancer Observatory, in 2020 were estimated more than 2.2 million new cases and approximately 1.8 million deaths worldwide, and a continuously increasing trend for both the incidence and mortality rates is expected for the next 20 years [1]. The narrowness in the gap between 
incidence and mortality rates, witnesses the difficulties in the clinical management of patients with lung cancer, especially those diagnosed with advanced stage disease, and the persistence of high mortality rates in this subset of patients. It is currently estimated that less than $21 \%$ of lung cancer patients are alive after five years from diagnosis [2], and this depends on several factors, such as the silent clinical course of the disease that leads to a late diagnosis in most cases, advanced age, impaired lung function related with tobacco smoking, cardiovascular and other comorbidities, histological subtype of the disease, and others [3, 4]. Significant improvements in lung cancer survival have been obtained in the last decade with the introduction of two novel therapeutic approaches for patients affected by non-small cell lung cancer (NSCLC), a group of histological subtypes that includes approximately $85 \%$ of lung cancers: immunotherapy and gene targeted therapy.

Immunotherapy with immune check point inhibitors (ICIs) is currently available for all programmed death 1 (PD1)/programmed death ligand 1 (PDL1) positive NSCLC subtypes, while targeted therapies are limited to patients with lung adenocarcinoma harboring specific genetic alterations like EGFR, BRAF, and MET mutations, as well as $A L K, R O S 1$, and $R E T$ rearrangements or NRTK1/2/3 gene fusions [5].

Tyrosine Kinase Inhibitors (TKIs) against mutations of the EGFR gene were the first to introduce survival benefits in patients with lung adenocarcinoma, opening a new era in this setting. Several clinical trials reported improved outcomes with first (i.e. gefitinib, erlotinib) and second (i.e. afatinib, dacomitinib) generation anti-EGFR TKIs, which however were time-limited because of the occurrence of resistance against these drugs, especially in patients with tumors harboring specific mutations, like the T790M mutation in exon 20 of the EGFR gene [6]. Currently a third generation anti-EGFR TKI, which overcomes most (but not all) the known resistances, is available (Osimertinib). Survival improvements have been mainly documented in clinical trials designed to investigate specific drugs or treatment combinations, but less is known from real-life studies investigating the prognostic impact in daily practice-selected patients. In addition, mutations in other genes like KRAS, BRAF or HER2 have been reported to be negative prognostic biomarkers in patients with lung adenocarcinoma, making the prognostic landscape more complex [7]. In the present study we investigated the global survival rates in a cohort of Sardinian patients with lung adenocarcinoma harboring driver genetic alterations in the EGFR, KRAS, BRAF, ALK and $M E T$ genes with the aim to examine their real-life impact on survival, and potential correlations with several demographic, life-habit and clinical factors.

\section{Materials and methods}

\section{Patients}

A series of consecutive Sardinian patients with a histologically proven diagnosis of locally advanced or metastatic lung adenocarcinoma who underwent genetic molecular testing were retrospectively enrolled from January 2011 through July 2016. The demographic and clinical data at the time of diagnosis were retrieved from medical records and pathology reports. Survival data were retrieved from the Cancer Registry of the Province of Sassari, which makes part of a wider web of tumor registries coordinated by the Italian Association for Tumor Registries (Associazione Italiana Registri Tumori, AIRTUM) [4]. Sardinian origin was ascertained through verification of the place of birth for all patients. All patients were informed about the aims of this study and, before the tissue sample was collected, provided written informed consent. The study was performed in accordance with the principles of the Declaration of Helsinki and was approved by the Committee for the Ethics of the Research and Bioethics of the National Research Council (CNR).

\section{Molecular testing}

For molecular testing, formalin-fixed paraffin-embedded (FFPE) tissue sections containing at least $80 \%$ of malignant cells from each tumor were obtained; in cases with lower neoplastic cell content, tissue sections underwent tumor macro-dissection using a single edge razor blade and a marked haematoxylin/eosin slide as a guide to remove unwanted tissue parts. All samples were processed at the Unit of Cancer Genetics of the National Research Council in Sassari (Italy), which performed routine molecular testing for all the Sardinian hospitals in the period of the study.

The molecular tests to perform were based on the clinical needs of each single case (mutations in exons 18, 19, and 21 of $E G F R$ as firstly-required test; immediately afterwards, $A L K$ rearrangements), and the availability of tissue to submit in further analysis of three genes not used in clinical practice for targeted therapies at the time of the study (mutations in all coding exons of KRAS and exon 15 of $B R A F ; M E T$ rearrangements), but with active involvement in the pathogenesis of lung cancer. Overall, all patients who were screened for other driver mutations in addition to the EGFR ones underwent genetic analysis for all the remaining genes (KRAS, ALK, BRAF, MET) independently on the positive or negative result by the EGFR mutation testing.

In our series, EGFR-mutated patients were treated with first (Gefitinib, Erlotinib) or second generation (Afatinib) anti-EGFR TKI, whose duration of efficacy varied due to the acquisition of drug resistance, mainly based on 
occurrence of the T790M mutation in exon 20 of the EGFR gene (data not shown; Casula et al., manuscript in preparation).

For mutation analysis genomic DNA was isolated from tissue sections using a standard protocol, and DNA quality was assessed for each specimen, as previously described [8]. Briefly, paraffin was removed from formalin-fixed paraffin-embedded (FFPE) samples by treatment with Bio-Clear (Bio-Optica, Milan, Italy), and DNA was purified using the GeneRead DNA FFPE Kit, (Qiagen Inc., Valencia, CA, USA) following the manufacturer's instructions. Yields of purified DNA were assessed by the Qubit dsDNA High-Sensitivity Assay Kit on the Qubit 2.0 Fluorometer (Life Thermofisher, Waltham, MA USA). Mutation analysis was conducted in the coding sequence of the following genes: EGFR (exons 18, 19, and 21), KRAS (exons 2, 3, and 4), and BRAF (exon 15). Quantitative measurements of mutations were based on pyrosequencing performed on a Pyro- Mark Q24 system (Qiagen Inc., USA) with a detection limit of 5-7\%, following the manufacturer's instructions [9]

Next-generation sequencing (NGS) was performed in 193 FFPE tissues using Ion S5-GeneStudio System and carried out by Ion Oncomine ${ }^{\mathrm{TM}}$ Focus Gene Assay which provides multiplexed target selection of 35 hotspot genes implicated in cancer research. Starting DNA and libraries were accurately quantified using a fluorescencebased quantification method, such as Qubit dsDNA HS. Result filtering, annotation, and data analysis workflow was performed by automated data transfer, from the Ion Torrent ${ }^{\mathrm{TM}}$ Server to the Ion Reporter Server for variant analysis. Coverage of $>300$ reads and frequency of mutated alleles $>3 \%$ for gene amplicon, in order to get a total amount of at least 10 mutated alleles for each candidate amplicon, were usually adopted for mutation selection criteria at somatic level. To even verify the existence at baseline of tumor subclones carrying the EGFR-T790M variant, we arbitrarily decided to go below the above-mentioned threshold value in order to identify up to few mutated alleles-ranging from $3(0.15 \%)$ to 28 (1.47\%) EGFR-T790M variants with a coverage of about 1900 reads-using the Integrative Genomics Viewer (IGV) tool.

Fluorescence in situ hybridization (FISH) for $M E T$, was carried out in interphase tumor cells using the specific CTB.13 N12 BAC probe (at the 7q31.2 locus) and the control centromere, labeled with Spectrum-Orange and Spectrum-Green (Vysis, Downer's Grove, IL, USA), respectively. For $A L K$, we used the ALK Break Apart FISH Probe Kit (Vysis, USA), as the first methodology deployed widely according to the recommendations by the National Comprehensive Cancer Network/NCCN guidelines (Version 3.2011). For $A L K$, the presence of rearrangement was defined when $\geq 15 \%$ of cells were positive for FISH signals according to the indications provided for the ALK Break Apart FISH Probe Kit (Vysis, USA). Amplification of the MET gene was defined by the presence of at least one of the following: (a) candidate gene to control centromere ratio $\geq 2$, according to the main criterion provided for assessing EGFR gene copy number in NSCLC; and/ or (b) presence of at least a tetrasomic signal ( $\geq 2.0$ gene copies per control centromere) in more than $15 \%$ of cells.

\section{Statistical analysis}

Descriptive analysis for qualitative and quantitative variables was conducted using proportions and the mean \pm standard deviation (SD) or median and interquartile range (IQR), respectively. Statistical differences between groups were compared using unpaired Student's $t$-test, Mann-Whitney rank sum test, chi-square test or Fisher's exact test as appropriate. Survival was investigated with Kaplan-Meier and Cox regression analyses. $p \leq 0.05$ was considered statistically significant. Data were analyzed using MedCalc for MS Windows, version 15.4 64 bit (MedCalc Software, Ostend, Belgium).

\section{Results}

A total of 1440 patients with lung adenocarcinoma who underwent genetic test to establish the presence of targeted genetic alterations in the period under investigation were identified. Among them, 158 were excluded because of lack of detailed clinical and/or follow-up data. Finally, 1282 patients were enrolled. Table 1 summarizes the global demographic, clinical and mutational data of the patients enrolled.

EGFR mutation analysis was performed in all the 1282 cases, as it was the first to be introduced in clinical practice, while KRAS and BRAF testing was carried out in 944 cases with tissues available to analyze. As stated in Methods, all patients with available tumor tissue sample were screened for mutations in the entire series of additional candidate genes (KRAS, ALK, BRAF, MET) regardless of the positive or negative result of the EGFR mutation testing.

The study of $A L K$ rearrangements started with the adoption of the test in clinical practice in 2012 and involved 880 patients, and since then also $M E T$ amplifications were searched in 692 cases with available samples. Eight hundred forty-eight (66\%) of the patients enrolled were males, and the median age was 67 (IQR 60-73) years (Table 1). EGFR, KRAS, and BRAF mutations were detected in $176(13.7 \%), 201(21.3 \%)$, and 28 (3\%) of tested cases, respectively. ALK rearrangements and $M E T$ amplifications were found respectively in $41(4.7 \%)$ and $14(2 \%)$ of tested cases. In two cases, 
Table 1 Demographic, clinical and mutational data of the patients included in the study

\begin{tabular}{|c|c|}
\hline Total cases & 1282 \\
\hline Age, median (IQR) & $67(60-73)$ \\
\hline$\leq 50, \mathrm{n}(\%)$ & $100(8.5)$ \\
\hline$>50, \mathrm{n}(\%)$ & $1182(91.5)$ \\
\hline Males, n (\%) & $848(66)$ \\
\hline Never smokers/ data available, n (\%) & $140 / 780(17.9)$ \\
\hline EGFR mutated/ analyzed (\%) & $176 / 1282(13.7)$ \\
\hline Exon 18, n (\%) & $11(6.2)$ \\
\hline Exon 19, n (\%) & $89(50)$ \\
\hline Exon 21, n (\%) & $78(43.8)$ \\
\hline KRAS mutated/analyzed (\%) & $201 / 944(21.3)$ \\
\hline Exon 2, n (\%) & $171(85.1)$ \\
\hline G12C & $68(39.8)$ \\
\hline G12V & $38(22.2)$ \\
\hline G12D & $34(19.9)$ \\
\hline Other G12 & $21(12.3)$ \\
\hline G13D & $10(5.8)$ \\
\hline Exon 3, n (\%) & $30(14.9)$ \\
\hline Q61H & $15(50)$ \\
\hline Q61L & $15(50)$ \\
\hline BRAF V600E mutated/analyzed (\%) & 28/944 (3) \\
\hline$A L K$ rearranged/analyzed (\%) & $41 / 880(4.7)$ \\
\hline MET amplified/analyzed (\%) & $14 / 692(2)$ \\
\hline \multicolumn{2}{|l|}{ Concomitant genetic alterations } \\
\hline$E G F R+M E T, n(\%)$ & $2(0.3)$ \\
\hline$A L K+M E T, \mathrm{n}(\%)$ & $2(0.3)$ \\
\hline Follow-up time, mean $( \pm S D)$, months & $46.1( \pm 27.4)$ \\
\hline Survival, mean $( \pm S D)$, months & $19.9( \pm 22.4)$ \\
\hline
\end{tabular}

EGFR simultaneous mutations in different exons were observed; only rare concomitant genetic alterations in different genes were observed (Table 1). The exons affected in each single mutated gene are summarized in Table 1. Further details on the specific subtypes of the genetic alterations found and their correlation with demographic, pathologic and smoking exposure data have been previously published in a larger series partially containing the current cases [10].

EGFR mutations were significantly more common in females and never smokers, as opposed to KRAS mutations which were significantly more frequent in males and ever smokers (Table 2). $A L K$ rearrangements were more common in younger patients, with the difference being close to statistical significance. $B R A F$ mutations were more common in males and smokers, but these differences did not reach statistical significance, while $M E T$ amplifications were significantly more frequent in females than in males (Table 2).

The mean $( \pm \mathrm{SD})$ follow-up time was 46.1 (27.4) months, and at the time of follow-up 994 (77.5\%) patients were dead. The mean $( \pm S D)$ survival in the global cohort was $19.9( \pm 22.4)$ months. Considering only dead patients, the mean $( \pm S D)$ overall survival was $15.8( \pm 18.8)$ months; a significantly greater survival was observed in females in comparison to males $(19 \pm 22.1$ vs $21.8 \pm 23$ months; $p=0.003)$ and never smokers in comparison to ever smokers $(26.4 \pm 23.7$ vs $21.1 \pm 22.5$ months; $p=0.003)$. Among patients with EGFR mutations, the eleven patients with exon 18 mutations had a significantly lower survival in comparison with those with exon 19 and exon 21 mutations $(8.8 \pm 5.7$ vs $24.9 \pm 20.9$ months; $p=0.0023)$; no such difference was detected between the latter or between patients with KRAS exon 2 and exon 3 mutations. Finally, 193 patients were tested for the EGFR-T790M mutation at baseline-before the beginning of the systemic treatment and independently on the result of the EGFR testing for TKI-sensitizing mutations-by a NGS assay; among them, 26 (13.5\%) had a very limited amount (very few mutated alleles) of the EGFR-T790M

Table 2 Genetic alterations in accordance with the gender, smoking habit, and age of the patients tested

\begin{tabular}{|c|c|c|c|c|c|c|c|c|}
\hline Males & Females & $p$ & Never smokers & Ever smokers & $p$ & Age $\leq \mathbf{5 0}$ & Age $>50$ & $p$ \\
\hline \multicolumn{9}{|l|}{$\operatorname{EGFR}(\mathrm{n}=1282)$} \\
\hline $62 / 848(7.3 \%)$ & $114 / 434(26.3)$ & $<0.0001$ & $62 / 140(44.3 \%)$ & $114 / 641(17.8 \%)$ & $<0.0001$ & 18/100 (18\%) & 158/1182 (13.4\%) & 0.2537 \\
\hline \multicolumn{9}{|l|}{$K R A S(\mathrm{n}=944)$} \\
\hline $156 / 619(25.2 \%)$ & 45/325 (13.8\%) & 0.0001 & $14 / 133(10.5 \%)$ & 187/585 (32\%) & $<0.0001$ & 18/83 (21.7\%) & $183 / 861(21.2 \%)$ & 0.9613 \\
\hline \multicolumn{9}{|l|}{$B R A F(n=944)$} \\
\hline 20/619 (3.2\%) & $8 / 325(2.5 \%)$ & 0.6453 & $2 / 133(1.5 \%)$ & 26/585 (4.4\%) & 0.1825 & $2 / 83(2.4 \%)$ & 26/861 (3\%) & 0.9794 \\
\hline \multicolumn{9}{|l|}{$A L K(\mathrm{n}=880)$} \\
\hline 26/592 (4.4\%) & 15/288 (5.2\%) & 0.7123 & $5 / 82(6.1 \%)$ & 36/398 (9\%) & 0.5140 & 6/57 (10.5\%) & $35 / 823(4.2 \%)$ & 0.0645 \\
\hline \multicolumn{9}{|l|}{$M E T(n=692)$} \\
\hline 5/471 (1\%) & 9/221 (4.1\%) & 0.0247 & $2 / 75$ (2.7\%) & $12 / 345$ (3.5\%) & 1.000 & 0/46 (0\%) & $14 / 646$ (2.1\%) & 0.6883 \\
\hline
\end{tabular}

Statistically significant values $(p<0.05)$ are evidenced in bold 
variant and showed a significantly lower survival in comparison to those without $(8.7$ vs. 47.4 months, $p<0.0001$ ).

Kaplan-Meier global survival estimates in accordance with the genetic alterations detected are depicted in Table 3.

Kaplan-Meier estimates showed that patients harboring EGFR mutations had a significantly longer survival in comparison to those without $(p<0.0001)$, as opposed to KRAS mutations, which was associated with a significantly lower survival $(p=0.0058)$. The Kaplan-Meyer survival curves of EGFR and KRAS mutated patients in

Table 3 Survival in accordance with the genetic alterations found in the study. Values are expressed in mean (confidence interval 95\%) months

\begin{tabular}{lllc}
\hline Gene & Altered & Wild type & $\boldsymbol{p}$ \\
\hline EGFR & $36.5(30.6-42.4)$ & $28.4(25.5-31.4)$ & $<\mathbf{0 . 0 0 0 1}$ \\
KRAS & $24.4(19.4-29.4)$ & $32.5(29.0-36.0)$ & $\mathbf{0 . 0 0 5 8}$ \\
BRAF & $32.5(17.3-47.7)$ & $30.7(27.6-33.8)$ & 0.7337 \\
ALK & $20.4(12.8-27.9)$ & $25.6(22.2-28.9)$ & 0.6493 \\
MET & $23.4(10.2-36.6)$ & $25.9(22.2-29.5)$ & 0.8454 \\
EGFR & & & \\
Males & $33.9(23.8-43.9)$ & $26.4(23.2-29.6)$ & 0.0729 \\
Females & $36.7(30.3-43.1)$ & $31.9(26.8-37.0)$ & $\mathbf{0 . 0 0 1 7}$ \\
Smokers & $33.1(21.6-44.6)$ & $26.4(23.0-29.9)$ & 0.1261 \\
Never smokers & $38.6(30.3-46.8)$ & $29.6(20.6-38.6)$ & $\mathbf{0 . 0 2 7 5}$ \\
KRAS & & & \\
Males & $23.4(18.7-28.1)$ & $29.1(25.2-33.1)$ & 0.3026 \\
Females & $24.2(12.7-35.6)$ & $36.5(31.4-41.7)$ & $\mathbf{0 . 0 0 2 5}$ \\
Smokers & $24.0(18.8-29.1)$ & $27.8(23.8-31.8)$ & 0.4366 \\
Never smokers & $13.1(6.7-19.4)$ & $34.7(27.8-41.5)$ & $\mathbf{0 . 0 0 5 5}$ \\
\hline
\end{tabular}

Statistically significant value is evidenced in bold comparison to those with wild-type tumors are depicted in Fig. 1, while Fig. 2 depicts the survival curves of patients with EGFR-T790M mutation in comparison to those without. EGFR mutations provide globally a significant survival advantage in females and never smokers as opposed to KRAS mutations, which were shown to be negative prognostic factors in the same subsets. No statistically significant differences in survival were found regarding the other genetic alterations investigated. In the Cox regression model summarized in Table 4, survival rates were evaluated after adjustment for the genetic alterations under investigation as well as for age, sex and smoking status; in this model EGFR mutations represented the only parameter, which impacted significantly on global survival.

\section{Discussion}

Our study evidenced that, among the driver genetic alterations evaluated, in patients with lung adenocarcinoma the main factor that positively impacts real-life survival is the presence of an EGFR mutation in exons 18, 19 or 21 , especially when associated with the absence of the T790M mutation in exon 20, no smoking history and female sex. This, in addition to the fact that EGFR mutations are the most common druggable genetic alterations in lung adenocarcinomas (10-16\% in Caucasians, up to $78 \%$ in Asians $[7,8,10])$, and the fact that our series does not include patients treated with the third generation TKI Osimertinib, confirm that anti-EGFR treatments represent the cornerstone of modern targeted therapies in this setting. Other studies showed better survival rates in females and never smokers $[11,12]$.

In addition, in our series patients with tumors with exon EGFR 18 mutations had a worse survival in comparison to those with exon 19 and 21 mutations, as previously described [13]. Of note, in our study, 26 patients
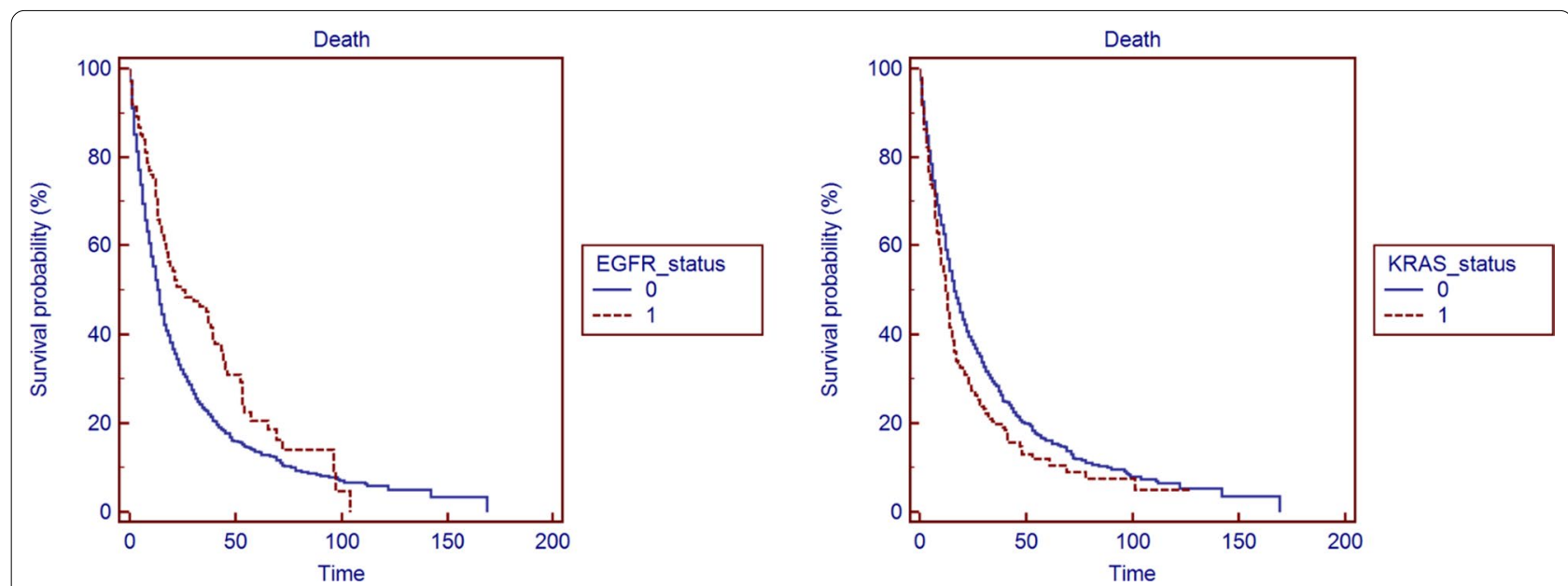

Fig. 1 Kaplan-Meyer survival curves in patients with and without EGFR and KRAS mutations, respectively. Time is referred to follow-up months 


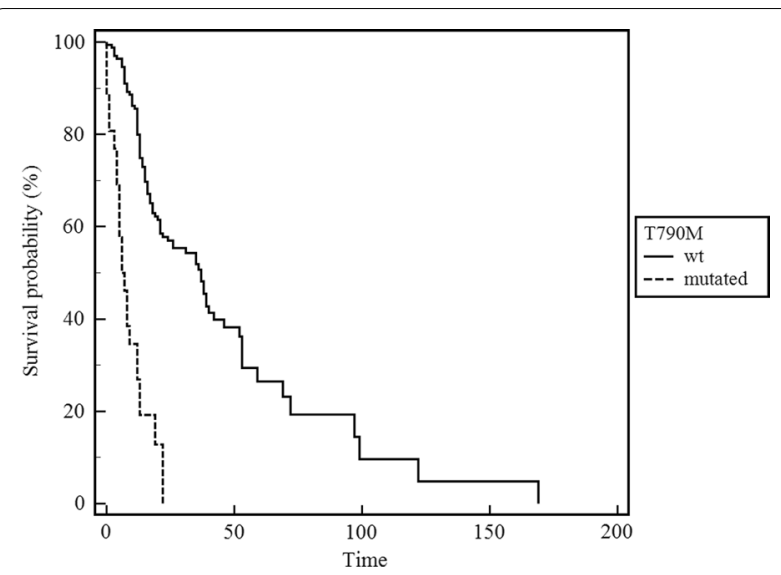

Fig. 2 Kaplan-Meyer survival curves in patients with and without EGFR-T790M mutation. Time is referred to follow-up months

Table 4 Cox regression analysis including the main impacting factors and the genetic alterations under investigation

\begin{tabular}{llll}
\hline Factor & HR & $\mathbf{9 5 \%} \mathbf{C l}$ & $\boldsymbol{p}$ \\
\hline Age & 0.9963 & $0.9846-1.0081$ & 0.5352 \\
Sex & 1.1872 & $0.9033-1.5604$ & 0.2184 \\
Smoking status & 1.0137 & $0.7085-1.4503$ & 0.9407 \\
EGFR & 0.6254 & $0.4497-0.9561$ & $\mathbf{0 . 0 2 8 3}$ \\
KRAS & 0.9448 & $0.7419-1.2032$ & 0.6453 \\
BRAF & 1.0292 & $0.5419-1.9546$ & 0.9300 \\
ALK & 0.9213 & $0.5966-1.4227$ & 0.7114 \\
MET & 0.8324 & $0.3904-1.7750$ & 0.6349 \\
\hline
\end{tabular}

Statistically significant value is evidenced in bold

(13.5\% of those tested) had tumors harboring the T790M mutation in the same tissue sample used for EGFR mutation analysis; despite a very few amount of mutated alleles being detected by NGS assay, such a subset of patients presented a consistently worse prognosis in comparison with cases who did not had the T790M variant. The EGFR-T790M mutation alone accounts for up to $50 \%$ of resistances in TKIs, which affect the majority of patients within the first year of treatment, and is considered quite rare at diagnosis, being the main mechanism of acquired (or secondary) drug resistance [14-16]. In this sense, our data show that, even if such a mutation is present at sub-clonal level in about one tenth of cases at the time of diagnosis, it may somehow affect the tumor behavior by causing intrinsic (or primary) drug resistance and inducing poor prognosis. This underlines the need of searching this mutation right from the initial molecular testing in order to both detect primary resistance to first and second generation TKIs and better classify the LAC patients from the prognostic point of view. Therefore, the occurrence of EGFR-T790M needs to be assessed despite the fact that last generation TKI drugs provide survival advantages in patients with early detected and persistent EGFR-T790M mutation [17]. Nevertheless, recent studies reported that the presence of EGFR-T790M in combination with complex EGFR mutations is a negative prognostic factor in patients treated with Osimertinib [18], suggesting that the need for EGFR-T790M testing will not come to an end with third generation TKIs. It appears, therefore, essential to use the biopsy or surgery samples to perform wider genetic testing that includes numerous EGFR variants and other genetic alterations which impact prognosis and survival, dependently or independently with the development of resistance to TKIs, like $A L K, K R A S, B R A F$ and $M E T$ alterations [6]. The advent of next generation sequencing (NGS) technologies allows wider genetic evaluations, but it is currently expensive and not immediately available, especially in centers with low case numbers. The last version (4-2021) of the National Comprehensive Cancer Network recommends, when feasible, testing be performed via a broad panel-based approach, and, if not available, provides recommendations for other testing methodologies [5].

KRAS mutations have been traditionally considered a negative prognostic factor in patients with lung adenocarcinoma [7], currently representing the biggest challenge for the modern precision oncology. This is because KRAS mutations are generally mutually exclusive with EGFR mutations, as in our series, despite the results of some studies, which found a certain degree of cooccurrence $[19,20]$. As a result, KRAS mutations generally affect consistent percentages of Caucasian (21-33\%, $21.3 \%$ in our series) and to a lesser extend Asian (2-15\%) patients with lung adenocarcinoma $[7,10]$, representing therefore an attractive target for future treatments. Several studies and a recent meta-analysis evidenced that $K R A S$ mutations have a worse prognosis and a reduced or absent response to EGFR TKIs [21]. However, other studies failed to show a per se negative prognostic impact of KRAS mutations in patients with lung adenocarcinomas [22]. In our study, KRAS mutations were associated with worst prognosis, especially when associated with tobacco smoking, but they were not confirmed as independent negative prognostic factor of survival in Cox regression analysis.

Similarly, BRAF mutations and $M E T$ amplifications did not show an independent prognostic role in our cohort. Nevertheless, these alterations are consistently rarer in comparison to KRAS and EGFR mutations, and probably larger numbers are necessary to better evaluate their prognostic role. In our series, patients with $B R A F$ and $M E T$ genetic alterations did not receive any targeted 
treatment because no such treatment were available at the time of the study, despite $B R A F$ was a well-established therapeutic target in other malignancies like melanoma [23]. Currently, Dabrafenib and Trametinib are recommended for patients with a BRAF-V600E mutation, which was the only mutation detected in all the 28 cases (3\% of the total tested) in our series [5]; this will surely improve the prognostic role of $B R A F$ mutations in lung cancer in the future. $M E T$ amplifications were detected only in 14 cases ( $2 \%$ of those tested) in our series, a percentage consistently low considering that $M E T$ amplifications have been extensively documented as a mechanism of acquired resistance in 5-22\% EGFR-mutated NSCLC upon therapeutic pressure with EGFR-TKIs; in addition, only two of these amplifications co-occurred with EGFR mutations, and thus, in patients subsequently treated with TKIs [24]. This suggests that, as opposed to the EGFR-T790M mutation, MET amplification is rarely present at the time of diagnosis and thus, it is a rare cause of primary resistance to TKIs. Currently drugs for MET exon 14 skipping mutations, which occur in $1-10 \%$ of the cases [24, 25], have been approved for clinical use (Capmatinib, Crizotinib, Tepotinib) [5], making these mutations and the study of acquired gene amplifications essential for the clinical management of the patients.

As opposed to $M E T$ alterations, $A L K$ fusions represent a well-established therapeutic target in lung cancer, as they are detected in 3-7\% of NSCLCs and have been associated with an absence of smoking, younger age, and adenocarcinoma histology [26]. In our study, $A L K$ rearrangements occurred in $4.7 \%$ of the cases tested with no particular predilection for age, sex or smoking habits; two of the rearrangements occurred in concomitance with $M E T$ amplification. Curiously, the median survival of $A L K$ wild type patients was higher, although not statistically significant, in comparison to those with $A L K$ rearrangement. Actually, we do not have a full knowledge of the clinical history of the systemic treatment among such a subset of patients; therefore, we are not sure whether this unexpected lower survival among cases with $A L K$ rearrangement might be somehow due to a real-life ineffectiveness of the first generation $A L K$ inhibitors. Further real-life studies are necessary to better evaluate this finding, and to extend it to more recent second line medications.

Our study has some limitations, mainly its retrospective design and molecular testing based primarily on clinical needs, which limited the availability of samples for testing all the driver genes in all the cases. In addition, no liquid biopsies have been included in the study, and thus data on acquired EGFR-T790M-related resistance are not available. On the other hand, our study includes globally a relevant number of daily-practice cases, offering a unique insight on the impact of the main driver genetic alterations on the prognosis of lung adenocarcinoma.

\section{Conclusions}

In conclusion, our results evidenced that the presence of EGFR mutations represent the most impacting, independent prognostic factor, regardless of the type of TKIs received and despite the occurrence of EGFR-T790M mutations in $13.5 \%$ of the cases tested-a percentage that appears higher than those reported in past studies though we classified positive cases using a very low threshold (at least 3 mutated alleles). Some of the genetic alterations (i.e. BRAF-V600 and KRAS-G12C) became recently druggable and this will certainly improve their role in improving overall survival. Data from future reallife studies based on a more comprehensive molecular classification at the time of diagnosis of the advanced disease are constantly necessary to better evaluate the real impact of specific genetic alterations and their corresponding targeted therapies in patients with lung cancer.

\section{Acknowledgements \\ The Sardinian Lung Cancer (SLC) Study Group includes the following mem- bers who participated as investigators in this study and should be considered as co-authors: Pietro Pirina, Alessandro Giuseppe Fois, Carlo Putzu (Azienda Ospedaliero-Universitaria, Sassari, Italy); Giorgio Astara, Mario Scartozzi (Azienda Ospedaliero-Universitaria, Cagliari, Italy); Anna Maria Carta, Giuseppe Porcu (Azienda Ospedaliera Brotzu, Cagliari, Italy); Gianfranco Bardino, Claudio Sini (Ospedale Olbia, Italy); Francesca Capelli, Maria Giuseppina Sarobba (Ospedale Zonchello, Nuoro, Italy).}

\section{Authors' contributions}

Conceptualization, AC, GiP; methodology, MaC, MCS, AM, MiC, GrP, MP, DS; formal analysis and investigation AM, MCS, PP, DM, GP; resources, VD, AC, DS, GS; statistical analysis, AZ; data curation, PP, VD, AC, GiP; writing-original draft preparation, PP, GP; writing-review and editing, PP, MaC, MCS, AM, MiC, GrP, MP, VD, AZ, DS, AC, GiP; funding acquisition, GiP. All authors read and approved the final manuscript.

\section{Funding}

This study was partially funded by the Fondazione AIRC "Programma di ricerca 5 per Mille 2018-ID\#21073". AC received research funds by "Fondo di Ateneo per la Ricerca 2020" of the University of Sassari, Italy.

\section{Availability of data and materials}

All data generated or analysed during this study are included in this published article and its supplementary information files.

\section{Declarations}

Ethics approval

This study was conducted according to the principles of the Declaration of Helsinki and has been approved by the Ethical Committee Commissione per l'Etica della Ricerca e la Bioetica of the National Research Council/CNR n. 12629.

Informed consent

Informed consent was obtained from all subjects involved in the study.

\section{Competing interest}

The authors declare no conflict of interest. 


\begin{abstract}
Author details
${ }^{1}$ Department of Medical, Surgical and Experimental Sciences, University of Sassari, 07100 Sassari, Italy. ${ }^{2}$ Unit of Cancer Genetics, Institute of Biomolecular Chemistry (ICB), National Research Council (CNR), 07100 Sassari, Italy. ${ }^{3}$ Unit of Cancer Genetics, Institute of Genetic and Biomedical Research (IRGB), National Research Council (CNR), Traversa La Crucca 3, 07100 Sassari, Italy. ${ }^{4}$ Department of Biomedical Sciences, University of Sassari, 07100 Sassari, Italy. ${ }^{5}$ Medical Oncology, Civil Hospital, 07041 Alghero (SS), Italy.
\end{abstract}

Received: 7 September 2021 Accepted: 14 December 2021

Published online: 10 January 2022

\section{References}

1. https://gco.iarc.fr. Accessed on 02July 2021.

2. Lu T, Yang X, Huang Y, Zhao M, Li M, Ma K, et al. Trends in the incidence, treatment, and survival of patients with lung cancer in the last four decades. Cancer Manag Res. 2019;21(11):943-53.

3. Dela Cruz CS, Tanoue LT, Matthay RA. Lung cancer: epidemiology, etiology, and prevention. Clin Chest Med. 2011;32(4):605-44.

4. Paliogiannis P, Attene F, Cossu A, Budroni M, Cesaraccio R, Tanda F, et al. Lung cancer epidemiology in North Sardinia, Italy. Multidiscip Respir Med. 2013:8(1):45.

5. https://www.nccn.org/professionals/physician_gls/default.aspx. Accessed 04 July 2021.

6. Wang F, Diao XY, Zhang X, Shao Q, Feng YF, An X, et al. Identification of genetic alterations associated with primary resistance to EGFR-TKIs in advanced non-small-cell lung cancer patients with EGFR sensitive mutations. Cancer Commun (Lond). 2019;39(1):7.

7. Fois SS, Paliogiannis P, Zinellu A, Fois AG, Cossu A, Palmieri G. Molecular epidemiology of the main druggable genetic alterations in non-small cell lung cancer. Int J Mol Sci. 2021;22(2):612

8. Paliogiannis P, Attene F, Cossu A, Defraia E, Porcu G, Carta A, et al. Impact of tissue type and content of neoplastic cells of samples on the quality of epidermal growth factor receptor mutation analysis among patients with lung adenocarcinoma. Mol Med Rep. 2015;12(1):187-91.

9. Ihle MA, Fassunke J, König K, Grünewald I, Schlaak M, Kreuzberg N, et al. Comparison of high resolution melting analysis, pyrosequencing, next generation sequencing and immune-histochemistry to conventional Sanger sequencing for the detection of p.V600E and non-p.V600E BRAF mutations. BMC Cancer. 2014;14:13.

10. Colombino M, Paliogiannis P, Cossu A, Santeufemia DA, Sardinian Lung Cancer (SLC) Study Group, Sini MC, et al. EGFR, KRAS, BRAF, ALK, and CMET genetic alterations in 1440 Sardinian patients with lung adenocarcinoma. BMC Pulm Med. 2019:19(1):209.

11. Zhang YL, Yuan JQ, Wang KF, Fu XH, Han XR, Threapleton D, et al. The prevalence of EGFR mutation in patients with non-small cell lung cancer: a systematic review and meta-analysis. Oncotarget. 2016;7(48):78985-93.

12. Chapman AM, Sun KY, Ruestow P, Cowan DM, Madl AK. Lung cancer mutation profile of EGFR, ALK, and KRAS: Meta-analysis and comparison of never and ever smokers. Lung Cancer. 2016;102:122-34.

13. Cheng C, Wang R, Li Y, Pan Y, Zhang Y, Li H, et al. EGFR Exon 18 mutations in east Asian patients with lung adenocarcinomas: a comprehensive investigation of prevalence, clinicopathologic characteristics and prognosis. Sci Rep. 2015;5:13959.

14. Miles B, Mackey JD. Epidermal growth factor receptor tyrosine kinase inhibitors and lung cancer: history, epidemiology, and market outlook. Cureus. 2021;13(2):13470.

15. Liu WJ, Du Y, Wen R, Yang M, Xu J. Drug resistance to targeted therapeutic strategies in non-small cell lung cancer. Pharmacol Ther. 2020;206:107438.

16. Jin $Y$, Bao H, Le $X$, Fan $X$, Tang $M$, Shi $X$, et al. Distinct co-acquired alterations and genomic evolution during TKI treatment in non-small-cell lung cancer patients with or without acquired T790M mutation. Oncogene. 2020;39(9):1846-59.

17. Zhao Z, Li L, Wang Z, Duan J, Bai H, Wang J. The status of the EGFR T790M mutation is associated with the clinical benefits of osimertinib treatment in non-small cell lung cancer patients: a meta-analysis. J Cancer. 2020:11(11):3106-13.
18. Lin YT, Tsai TH, Wu SG, Liu YN, Yu CJ, Shih JY. Complex EGFR mutations with secondary T790M mutation confer shorter osimertinib progressionfree survival and overall survival in advanced non-small cell lung cancer. Lung Cancer. 2020;145:1-9.

19. Shigematsu H, Lin L, Takahashi T, Nomura M, Suzuki M, Wistuba II, et al. Clinical and biological features associated with epidermal growth factor receptor gene mutations in lung cancers. J Natl Cancer Inst. 2005;97:339-46.

20. Scheffler M, Ihle MA, Hein R, Merkelbach-Bruse S, Scheel AH, Siemanowski J, et al. K-ras mutation subtypes in NSCLC and associated co-occuring mutations in other oncogenic pathways. J Thorac Oncol. 2019;14:606-16.

21. Linardou H, Dahabreh IJ, Kanaloupiti D, Siannis F, Bafaloukos D, Kosmidis $P$, et al. Assessment of somatic k-RAS mutations as a mechanism associated with resistance to EGFR-targeted agents: a systematic review and meta-analysis of studies in advanced non-small-cell lung cancer and metastatic colorectal cancer. Lancet Oncol. 2008;9:962-72.

22. Winton T, Livingston R, Johnson D, Rigas J, Johnston M, Butts C, et al. Vinorelbine plus cisplatin vs. observation in resected non-small-cell lung cancer. N Engl J Med. 2005;352:2589-97.

23. Sini MC, Doneddu V, Paliogiannis P, Casula M, Colombino M, Manca A, et al. Genetic alterations in main candidate genes during melanoma progression. Oncotarget. 2018;9(9):8531-41.

24. Song Z, Wang H, Yu Z, Lu P, Xu C, Chen G, et al. De novo MET amplification in Chinese patients with non-small-cell lung cancer and treatment efficacy with Crizotinib: a multicenter retrospective study. Clin Lung Cancer. 2019;20:e171-6.

25. Drilon A, Cappuzzo F, Ou SI, Camidge DR. Targeting MET in lung cancer: will expectations finally be MET? J Thorac Oncol. 2017;12:15-26.

26. Shaw AT, Yeap BY, Mino-Kenudson M, et al. Clinical features and outcome of patients with non-small-cell lung cancer who harbor EML4-ALK. J Clin Oncol. 2009:27:4247-53.

\section{Publisher's Note}

Springer Nature remains neutral with regard to jurisdictional claims in published maps and institutional affiliations.

Ready to submit your research? Choose BMC and benefit from

- fast, convenient online submission

- thorough peer review by experienced researchers in your field

- rapid publication on acceptance

- support for research data, including large and complex data types

- gold Open Access which fosters wider collaboration and increased citations

- maximum visibility for your research: over $100 \mathrm{M}$ website views per year

At BMC, research is always in progress.

Learn more biomedcentral.com/submissions 